\title{
Prevalence of PALB2 mutations in Australian familial breast cancer cases and controls
}

\author{
Ella R. Thompson 1,2, Kylie L. Gorringe ${ }^{1,2,3}$, Simone M. Rowley ${ }^{1}$, Michelle W. Wong-Brown ${ }^{4}$, Simone Mclnerny ${ }^{5}$, \\ $\mathrm{Na} \mathrm{Li}{ }^{1,6}$, Alison H. Trainer ${ }^{1,5}$, Lisa Devereux ${ }^{1,7}$, Maria A. Doyle ${ }^{8}$, Jason Li ${ }^{8}$, Richard Lupat ${ }^{8}$, Martin B. Delatycki ${ }^{9}$, \\ LifePool Investigators, Gillian Mitchell ${ }^{2,5,10}$, Paul A. James ${ }^{2,3,5}$, Rodney J. Scott ${ }^{6,11}$ and lan G. Campbell ${ }^{1,2,3^{*}}$
}

\begin{abstract}
Introduction: PALB2 is emerging as a high-penetrance breast cancer predisposition gene in the order of BRCA1 and $B R C A 2$. However, large studies that have evaluated the full gene rather than just the most common variants in both cases and controls are required before all truncating variants can be included in familial breast cancer variant testing.

Methods: In this study we analyse almost 2000 breast cancer cases sourced from individuals referred to familial cancer clinics, thus representing typical cases presenting in clinical practice. These cases were compared to a similar number of population-based cancer-free controls.

Results: We identified a significant excess of truncating variants in cases (1.3\%) versus controls (0.2\%), including six novel variants ( $p=0.0001$; odds ratio (OR) 6.58, $95 \%$ confidence interval (CI) 2.3-18.9). Three of the four control individuals carrying truncating variants had at least one relative with breast cancer. There was no excess of missense variants in cases overall, but the common c.1676A > G variant (rs152451) was significantly enriched in cases and may represent a low-penetrance polymorphism ( $p=0.002$; OR 1.24 (95 \% Cl 1.09-1.47).

Conclusions: Our findings support truncating variants in PALB2 as high-penetrance breast cancer susceptibility alleles, and suggest that a common missense variant may also lead to a low level of increased breast cancer risk.
\end{abstract}

\section{Introduction}

Partner and localizer of BRCA2 (PALB2) plays a central role in homologous recombination-mediated repair of double-strand DNA breaks [1] and biallelic mutations are responsible for Fanconi anemia complementation group $\mathrm{N}$ [2]. Monoallelic inactivating germline mutations in PALB2 were subsequently shown to be associated with familial breast cancer [3] and numerous studies supported this association in various populations and established a mutation prevalence of approximately $1 \%$ among familial breast cancer cases (varying from 0.1 $\%$ to $2.7 \%$ as reviewed by Southey et al. [4]). Most recently, Antoniou et al. used a modified segregation analysis approach to determine that the age-specific risk of

\footnotetext{
* Correspondence: ian.campbell@petermac.org

'Cancer Genetics Laboratory, Peter MacCallum Cancer Centre, Locked Bag 1,

A'Beckett St, East Melbourne, VIC 8006, Australia

${ }^{2}$ The Sir Peter MacCallum Department of Oncology, St Andrews Place, East Melbourne, VIC 3002, Australia

Full list of author information is available at the end of the article
}

breast cancer among female mutation carriers overlaps the risk conferred by $B R C A 2$ mutations [5] establishing that, despite the rarity of mutations, PALB2 is the most significant breast cancer predisposition gene after $B R C A 1$ and BRCA2.

In Australia, early studies identified PALB2 c.3113G > A (p.Trp1038*) as a recurring truncating mutation among familial breast cancer index cases, and established the enrichment of c.3113G > A in cases compared to controls [6]. Further studies have identified a spectrum of truncating variants among breast cancer cases [7-10], the collective frequency of which has not been compared to Australian controls. Indeed few studies of PALB2 mutations have analysed significant numbers of family cancer clinic-ascertained cases or matched controls. Because early studies focused on screening just for the presumed common pathogenic mutations, in Australia (eviQ Cancer Treatments Online; [11]) it is not recommended to test for $P A L B 2$ truncating mutations aside from the recurring c.3113G > A variant, however, it is likely that all truncating 
mutations confer an equivalent loss of gene function and consequent breast cancer risk. Other guidelines, such as National Comprehensive Cancer Network [12], have made no specific distinction between different PALB2 mutations but do raise a general caution around the interpretation of testing for mutations in PALB2 and other "moderate penetrance" breast cancer predisposition genes, especially as part of panel tests. Identification of genetic risk factors is critical for individual risk assessment and reduction strategies, and in the near future may provide avenues for personalised therapy [4]. Therefore it is important to continue to amass the necessary data to support the implementation of whole gene testing of PALB2 in breast cancer families. In this study, we performed germline mutation analysis of the entire coding region of PALB2 in a cohort of 1996 breast cancer index cases referred to familial cancer clinics for genetic testing and tested negative for BRCA1 and BRCA2 mutations as well as 1998 Australian cancer-free female controls. This represents the largest single case/control screen of germline PALB2 mutations to date.

\section{Methods}

\section{Samples for mutation analysis}

Cancer-affected women in the study were referred by their physician to a specialist Familial Cancer Centre (FCC) for genetic testing of BRCA1 and BRCA2 between 1997 and 2014, and were identified as being at "high risk" of carrying a predisposing allele. The criteria for high risk included a personal history of breast cancer, two or more first- or second-degree relatives with breast and/or ovarian cancer, and an additional risk factor (additional affected close relatives, diagnosis before 40 years, multiple primary breast or ovarian cancers in one individual, or Ashkenazi Jewish ancestry). From 2003, individuals with $\mathrm{a} \geq 10 \%$ risk of carrying a $B R C A 1$ or $B R C A 2$ mutation, as estimated by BRCAPro, including tumour pathology, were also eligible [13].

Our final case cohort (Additional file 1) included 997 breast (95\%) or ovarian (5\%) cancer-affected index cases from the Hunter Area Pathology Service (HAPS), Newcastle, Australia [9]. Family history information was available for a subset of this cohort only. A further 999 breast cancer-affected index cases each with detailed family history available were obtained from the combined Victorian Familial Cancer Centres (FCCs) through the Variants in Practice (ViP) study. For all cases, clinical genetic testing of $B R C A 1$ and $B R C A 2$, including for large rearrangements by multiplex ligation probe-dependent amplification (MLPA), returned negative results.

A cohort of 1998 participants in the LifePool study [14] were utilised as cancer-free population control samples for this analysis. LifePool recruits female participants through the Australian population mammography screening program (BreastScreen) for research studies utilising prospectively collected epidemiological, genetic and mammographic data with ongoing clinical follow-up obtained through the Victorian Cancer Registry. Participants provided breast cancer family history information for close relatives only. The average age of the participants recruited to this study was $58.84 \pm 9.9$ years (range 19-91).

All cases and controls provided informed consent for genetic analysis of their germline DNA. This study was approved by the Human Research Ethics Committees at each participating ViP centre (see Acknowledgements), the Peter MacCallum Cancer Centre, Hunter New England Health and The University of Newcastle. This study was carried out in accordance with all relevant regulations and guidelines.

\section{Germline mutation analysis}

Germline mutation analysis of the PALB2 gene was performed as part of a custom sequencing panel. All coding PALB2 exons were amplified from $225 \mathrm{ng}$ of germline DNA extracted from blood or saliva using the HaloPlex Targeted Enrichment Assay (Agilent Technologies, Santa Clara, CA, USA) according to the manufacturer's protocol using an Agilent Bravo Automated Liquid Handling System. Paired-end 100 or 150 bp sequence reads were generated from the indexed, pooled libraries on a HiSeq2500 Genome Analyzer (Illumina, San Diego, CA, USA). Sequence reads were trimmed of adapter using Cutadapt [15] and aligned using either BWA or BWA MEM [16]. Genome Analysis Toolkit (GATK) v3.1 was used to perform indel realignment and Unified Genotyper was used for variant calling $[17,18]$. Protein consequence and additional annotations were added using Ensembl v73 Variant Effect Predictor [19]. Variant positions were determined by reference to GenBank reference sequence NM_024675.3 according to Human Genome Variation Society (HGVS) guidelines [20]. All novel variants were validated by Sanger resequencing of germline DNA using primers from Tischkowitz et al. [21]. The following in silico prediction tools were used to assess the possible pathogenicity of missense mutations: Combined Annotation-Dependent Depletion (CADD) [22], Condel [23], SIFT [24] and PolyPhen2 [25]. CADD scores evaluate both missense and indel variants, integrating conservation measures, regulatory, transcriptional and protein effects to estimate the relative deleteriousness of the variants.

\section{Results \\ Coverage}

A total of 1996 breast cancer index cases and 1998 noncancer controls were screened for germline mutations in the coding regions of $P A L B 2$. These coding regions were well covered by sequence reads in both cases and controls. 
The mean read depth across the entire gene for all samples was 217 (192 for cases, 242 for controls), with an average of $98.66 \%$ of the coding regions covered by at least 20 reads ( $98.12 \%$ for cases and $99.20 \%$ for controls).

\section{Truncating mutations}

Nineteen different truncating variants were identified in 30 individuals in this study, 26 of these variants were detected among 1996 breast cancer index cases (1.3 \%) and four among 1998 controls (0.2\%), demonstrating a significant enrichment in cases $(p=0.0001$, chi-squared test; odds ratio (OR) 6.58, 95 \% confidence interval (CI) 2.3-18.9) (Table 1). Five previously reported variants were detected recurrently (c.196C $>\mathrm{T}$ (p.Gln66*) and c.758dupT (p.Ser254Illefs*3) each in two cases, c.3113G > A (p.Trp1038*) in seven cases and one control, and c.3116delA (p.Asn1039Ilefs*2) and c.3362delG (p.Gly1 121 Valfs*3) each in one case and one control) with the remaining variants detected in single cases or controls only. Six truncating variants have not previously been reported (c.522_523delAA (p.Arg175Thrfs*9), c.577dupA (p.Thr193Asnfs*2), c.693dupA (p.Gly232Argfs*3), c.86 OdupT (p.Ser288Lysfs*15), c.1947_1966dup (p.Pro65
6Glnfs"11) and c.2966_2967insCAACAAGT (p.Glu99 0Asnfs*3)). Novel variant p.Glu990Asnfs*3 was detected in a control only.

The personal and family history information for carriers of the PALB2 truncating variants are given in Table 2 and Additional file 2. As expected, the cases generally have a strong family history of cancer, especially breast cancer. In the controls, four individuals were identified with truncating variants. One individual had a maternal aunt diagnosed with breast cancer at under 40 years of age, and her mother, father and brother all had cancer although not of the breast. The mothers of both of the other individuals had a breast cancer diagnosis aged over 70 years of age, and one of these individuals also had two second-degree relatives with breast cancer. The final and youngest carrier (aged 48) did not report any breast cancer in her family. Thus, 3/4 carriers have some family history of breast cancer.

\section{Missense and synonymous variants}

A large number of missense variants $(n=54)$ were detected in the cohort (Table 3). There was a slight

Table 1 Truncating variants

\begin{tabular}{|c|c|c|c|c|c|c|c|c|}
\hline Exon & Nucleotide change $^{a}$ & $\begin{array}{l}\text { Predicted protein } \\
\text { change }^{\mathrm{a}}\end{array}$ & dbSNP ID & First reported & $\begin{array}{l}\text { HAPS cases } \\
(n=997)^{b}\end{array}$ & $\begin{array}{l}\text { FCC-ViP cases } \\
(n=999)^{b}\end{array}$ & $\begin{array}{l}\text { Controls } \\
(n=1998)^{b}\end{array}$ & $\begin{array}{l}\text { CADD scaled } \\
\text { C score }\end{array}$ \\
\hline 3 & c.172_175delTTGT & p.Gln60Argfs*7 & . & Jones (2009) & - & 1 & - & 19.0 \\
\hline 3 & c. $196 C>T$ & p.Gln66* & rs180177083 & Casadei (2011) [29] & 2 & - & - & 35.0 \\
\hline 4 & c.522_523delAA & p.Arg175Thrfs*9 & . & - & - & 1 & - & 23.2 \\
\hline 4 & c.577dupA & p.Thr193Asnfs*2 & . & - & 1 & - & - & 11.6 \\
\hline 4 & c.693dupA & p.Gly232Argfs*3 & . & - & 1 & - & - & 11.2 \\
\hline 4 & c.758dupT & p.Ser254llefs*3 & . & Zheng (2012) [40] & 1 & 1 & - & 16.2 \\
\hline 4 & c.860dupT & p.Ser288Lysfs*15 & . & - & 1 & - & - & 17.6 \\
\hline 5 & c.1947dupA & p.Glu650Argfs*13 & . & Teo (2013) [8] & - & 1 & - & 24.1 \\
\hline 5 & c.1947_1966dup & p.Pro656Glnfs*11 & . & - & 1 & - & - & 13.0 \\
\hline 5 & c. $2386 \mathrm{G}>\mathrm{T}$ & p.Gly796* & rs180177112 & Rahman (2007) [3] & - & 1 & - & 32.0 \\
\hline 5 & c.2391delA & p.Gln797Hisfs*54 & . & Wong-Brown (2013) & 1 & - & - & 23.5 \\
\hline 9 & c.2966_2967insCAACAAGT & p.Glu990Asnfs*3 & . & - & - & - & 1 & 20.7 \\
\hline 9 & c.2982dupT & p.Ala995Cysfs* 16 & rs180177127 & Rahman (2007) [3] & 1 & - & - & 31.0 \\
\hline 10 & $c .3113 G>A$ & p.Trp1038*c & rs180177132 & Rahman (2007) [3] & 2 & 5 & 1 & 42.0 \\
\hline 11 & c.3116delA & p.Asn 1039llefs*2 & rs180177133 & $\begin{array}{l}\text { Reid (2007) [2]; Rahman } \\
\text { (2007) [3] }\end{array}$ & - & 1 & 1 & 40.0 \\
\hline 12 & c. $3256 C>T$ & p.Arg1086* & . & Jones (2009) & - & 1 & - & 40.0 \\
\hline 13 & c.3362delG & p.Gly1121Valfs*3 & . & Blanco (2013) [27] & - & 1 & 1 & 22.1 \\
\hline 13 & c.3507_3508del & p.His1170Phefs*19 & . & Antoniou (2014) [5] & 1 & - & - & 40.0 \\
\hline 13 & c.3549C > G & p.Tyr1183* & rs118203998 & Reid (2007) [2] & 1 & - & - & 37.0 \\
\hline
\end{tabular}

HAPS Hunter Area Pathology Service, FCC Familial Cancer Centre, ViP Variants in Practice

${ }^{a}$ Variant positions are reported in reference to NCBI RefSeq NM_024675.3 (mRNA) and NP_078951.2 (protein)

${ }^{\mathrm{b}}$ Number of individuals carrying the variant

${ }^{c} \mathrm{c} .3113 \mathrm{G}>\mathrm{A}$ produces three different PALB2 mRNA sequences: complete deletion of exon 10 (117 bp); use of an alternative splice site within exon 10, and deletion of $31 \mathrm{bp}$; and an immediate stop at codon 1038 [29] 
Table 2 Family history of carriers of truncating variants

\begin{tabular}{|c|c|c|c|c|}
\hline Case & Variant $^{a}$ & Diagnosis & Family history first-degree relatives & Family history other relatives \\
\hline HAPS-102285 & p.Gln66* & Breast 41 & NA & NA \\
\hline HAPS-60382 & p.Gln66* & Breast 43 & Mother breast 61; father prostate 65 & $\begin{array}{l}\text { Paternal: } 2 \times 2^{\text {nd }} \text { cousins breast } \\
42,37 ; 3 \times \text { great aunt breast } 42, \\
47,58 \text {; grandfather prostate } 71 . \\
\text { Maternal: grandmother other } 60\end{array}$ \\
\hline HAPS-90978 & p.Thr193Asnfs*2 & Bilateral breast 61 & Sister breast $60 \mathrm{~s}$; sister breast $60 \mathrm{~s}$ & \\
\hline HAPS-90809 & p.Gly232Argfs*3 & Bilateral breast 41,47 & Father lung 68 & $\begin{array}{l}\text { Cousin breast } 45 \text {, maternal } \\
\text { grandmother bowel } 60\end{array}$ \\
\hline HAPS-120272 & p.Ser254llefs*3 & Breast 35, ovarian 58 & $\begin{array}{l}2 \times \text { Sisters breast } 40 \text { s; brother renal 52; } \\
\text { brother HNSCC } 50 \text { s; father other }\end{array}$ & Cousin breast 50 s. \\
\hline HAPS-102573 & p.Ser288Lysfs*15 & Breast 63 & NA & NA \\
\hline HAPS-114269 & p.Pro656Glnfs*11 & Breast 46, ovarian 49 & Mother unknown primary 45 & $\begin{array}{l}\text { Maternal aunt breast } 50 \text {; } \\
\text { grandmother breast } 60 \mathrm{~s} ; \\
\text { maternal uncle prostate } 70 \text { s }\end{array}$ \\
\hline HAPS-120953 & p.Gln797Hisfs*54 & Breast 46 & Father melanoma 62 & $\begin{array}{l}\text { half-brother NHL } 64 \text {; half-brother } \\
\text { bladder } 55 \text {; maternal cousin breast } \\
41 \text {; maternal aunt breast } 55\end{array}$ \\
\hline HAPS-81242 & p.Ala995Cysfs*16 & Bilateral breast 46,70 & $\begin{array}{l}\text { Sister breast 38; daughter breast 47; } \\
\text { daughter cervical 24; mother breast } 39\end{array}$ & $\begin{array}{l}\text { Paternal cousin breast } 60 \text { s; maternal } \\
\text { cousin other }\end{array}$ \\
\hline HAPS-121549 & p.Trp1038* & Breast 56 & Sister breast 44 & $\begin{array}{l}\text { Maternal aunt breast 50s; paternal } \\
\text { grandmother breast }\end{array}$ \\
\hline HAPS-110283 & p.Trp1038* & Breast 46 & Mother breast 57 & $\begin{array}{l}\text { Maternal: aunt bowel 50; } \\
\text { great- } \\
\text { grandmother ovarian. Paternal: } \\
\text { grandmother breast } 85 \text {, } \\
\text { grandfather bowel } 60\end{array}$ \\
\hline HAPS-100240 & p.His1170Phefs*19 & Breast 48 & Mother breast 68 & $\begin{array}{l}\text { Maternal grandmother AML 72; } \\
\text { paternal cousin ovarian } 36 .\end{array}$ \\
\hline HAPS-110583 & p.Tyr1183* & Breast 39 & $\begin{array}{l}\text { Mother breast 80; father } \\
\text { bowel } 69\end{array}$ & $\begin{array}{l}\text { Maternal aunt bilateral breast } \\
50,70 ; \text { maternal grandfather } \\
\text { prostate } 80\end{array}$ \\
\hline FCC-681-000 & p.Gln60Argfs*7 & Breast 47 & & Breast $>40$ \\
\hline FCC-2121-000 & p.Gly796* & Breast 62 & Lung/prostate & Prostate, other $\times 2$ \\
\hline FCC-1423-000 & p.Trp1038* & Breast 44 & Breast $>40$, bowel, other & Breast $>40$ \\
\hline FCC-2104-000 & p.Trp1038* & Breast 38 & Breast $>40$, other $\times 2$ & Other \\
\hline FCC-2677-000 & p.Trp1038* & $\begin{array}{l}\text { Cervix } 55 \text {, breast } 65 \text {, } \\
67,68 \text {, bowel } 67\end{array}$ & Breast $>40$, ovarian & Lung, other \\
\hline FCC-3527-000 & p.Trp1038* & Breast 37 & & Other \\
\hline FCC-60-000 & p.Trp1038* & $\begin{array}{l}\text { Thyroid } 42 \text {, breast } \\
48,51\end{array}$ & Breast $>40$, bowel & $\begin{array}{l}\text { Breast } \times 2 \text { ovarian, prostate } \times 2 \text {, } \\
\text { lung, other } \times 2\end{array}$ \\
\hline FCC-905-000 & p.Asn1039llefs*2 & $\begin{array}{l}\text { Melanoma 39, } \\
\text { breast } 47\end{array}$ & Lung, prostate, other & Breast $\times 3$, bowel $\times 2$, ovarian \\
\hline FCC-2965-000 & p.Arg1086* & Bilateral breast 44 & Prostate & \\
\hline FCC-317-000 & p.Gly1121Valfs*3 & Breast 31 & Other $\times 2$ & Breast, other \\
\hline FCC-1322-000 & p.Glu650Argfs*13 & Breast 50 & Breast, other & Breast $\times 3$, other \\
\hline FCC-3397-000 & p.Ser254llefs*3 & $\begin{array}{l}\text { Melanoma } 53 \\
\text { Breast } 54,61\end{array}$ & Breast & \\
\hline FCC_2431-000 & p.Arg175Thrfs*9 & Breast 42 & Breast, other $\times 3$ & Breast $\times 3$, lung, other $\times 2$ \\
\hline
\end{tabular}

\section{Controls}

LP-12031915

\section{Variant}

p.Trp1038*

\section{Age enrolled}

65
Family history first-degree relatives

Mother, other 58; father lung 64; brother other
Family history other relatives

Maternal aunt breast $>40$ 
Table 2 Family history of carriers of truncating variants (Continued)

\begin{tabular}{lllll}
\hline LP-13099711 & p.Asn1039llefs*2 & 73 & Mother breast 72 & $\begin{array}{l}\text { Maternal: grandmother } \\
\text { breast }>40 \text { yrs; aunt breast }\end{array}$ \\
LP-13243620 & p.Glu990Asnfs*3 & 73 & Mother breast 82 & NA \\
LP-12025195 & p.Gly1121Valfs*3 & 48 & None & Yes, no details \\
\hline
\end{tabular}

NA not applicable, HNSCC head and neck squamous cell carcinoma, NHL non-Hodgkin lymphoma

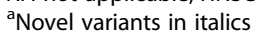

enrichment for missense variants overall in cases $(39.6 \%)$ versus controls $(36.1 \%, p=0.025$, OR 1.15 , $95 \%$ CI $1.02-$ 1.32). The four most common variants ( $>3 \%$ carrier frequency) were tested for association with breast cancer. Surprisingly, the most common variant, c.1676A > G (Gln559Arg; rs152451), which is predicted by CADD, Condel, PolyPhen2 and SIFT to be benign, was significantly more common in cases $(19.9 \%$ carried at least one non-wild-type allele) than controls (16.8 \% non-wild-type) with a per-allele OR of 1.24 (95 \% CI 1.09-1.47, $p=0.002$, logistic regression). There were 20 cases homozygous for this variant versus ten controls $(p=0.058$ logistic regression, OR 2.09, $95 \%$ CI 0.98-4.48). The overall trend for an effect had a $p$ value of 0.0018 (CochraneArmitage trend test). The minor allele frequency was 0.105 in cases and 0.086 in controls, compared to other databases where the minor allele frequency of this variant in European populations was 0.09 (1000 Genomes), 0.096 (ExAC) and 0.09 (EVS), but showed increased frequency in African and Asian populations.

Considering only those rare variants present in fewer than five carriers among 3994 cases and controls (approximately $0.1 \%$ ), a similar number of missense variants were detected in both groups (40 in cases (2\%), 28 in controls $(1.4 \%))$, which does not suggest any association of rare missense variants with risk. There was also no significant enrichment in cases when limited to rare variants that were predicted to be deleterious by any of Condel, SIFT or Polyphen2 (28/1996 cases, 18/1998 controls) or with a CADD score of $>10$ (29/1996 cases, 20/1998 controls).

We detected 23 synonymous variants (Table 4). Neither the most common alone (c.3300 $\mathrm{T}>\mathrm{G}$ ) nor all together were significantly enriched in cases or controls.

\section{Discussion}

This study screened Australian individuals with breast cancer who had been referred to a Familial Cancer Centre for genetic testing and in whom no pathogenic $B R C A 1$ and $B R C A 2$ variant could be identified. The frequency of PALB2 truncating variants in this cohort (1.1\%) is similar to other studies analysing high-risk breast cancer individuals $(0.64-3.4 \%, 1.35 \%$ overall $[3,6-9,26-41])$ or triple-negative breast cancer $(0.9-2.5 \%[10,42,43])$ but is the largest to include an analysis of the full gene in both cases and controls. However, we would not be able to detect any large deletions or rearrangements. The low frequency of truncating variants in controls supports PALB2 as a high-penetrance breast cancer predisposing gene. The diversity of truncating mutations identified, comprising 16 different variants in eight of the 13 exons including five novel variants, highlights the need for full gene screening, not just the most common variant c.3113G > A (rs180177132). These data will enable evidence-based clinical guidelines to include full PALB2 screening if previously they had advised testing limited to the specific common variant only.

The prevalence of truncating variants in cancer-free controls was $0.15 \%$ in the LifePool cohort. These individuals were ascertained from women attending populationbased mammographic screening, which in Australia is targeted towards women over 50, although some younger women are included. Thus, this volunteer cohort may not be entirely representative of the general population, although all were cancer-free at the time of analysis. Nonetheless, the frequencies of missense and synonymous variants are consistent with those reported in large databases such as 1000 Genomes [44], Exome Aggregation Consortium [45] and Exome Variant Server [46].

We did not observe any significant enrichment in missense mutations overall, although the frequency was slightly higher in the cases when only rare, deleterious mutations were considered. The contribution of rarer variants to breast cancer risk will need to be evaluated in larger case-control cohorts. Surprisingly, the common variant c.1676A > G (Gln559Arg; rs152451) was significantly enriched in cases versus controls, although with only a modest odds ratio (1.24). There was a trend towards homozygous carriers of this variant being enriched in cases versus controls with an OR of 2.08. This variant was shown to be associated with an increased breast cancer risk in multiple-case breast cancer families in Chile compared to population controls [47] with an OR of 2.0 when at least three family members were breast or ovarian cancer-affected. No association was found for individuals diagnosed at a young age $(<50)$ and with no affected relatives. In a small Malaysian case-control study, there was a trend towards enrichment for carriers of the variant in non-familial breast cancer cases $(286 / 871,33 \%)$ versus controls (70/257, $27 \%$, OR 1.3 [38]), however, cases and controls were not well matched for ethnicity, with an excess of Indian and Malay women over Chinese in the controls compared to cases. Larger numbers of cases and 
Table 3 Missense variants

\begin{tabular}{|c|c|c|c|c|c|c|c|c|c|c|c|c|c|c|}
\hline Exon & $\begin{array}{l}\text { Nucleotide } \\
\text { change }^{a}\end{array}$ & $\begin{array}{l}\text { Protein } \\
\text { change }^{\mathrm{a}}\end{array}$ & dbSNP ID & First reported & $\begin{array}{l}1000 \mathrm{G} \\
\mathrm{MAF}^{\mathrm{b}}\end{array}$ & $\begin{array}{l}\text { NHBLI GO } \\
\text { ESP MAF }^{c}\end{array}$ & $\operatorname{ExAC}^{d}$ & $\begin{array}{l}\text { HAPS cases } \\
(\mathrm{n}=997)^{\mathrm{e}}\end{array}$ & $\begin{array}{l}\text { FCC-ViP } \\
\text { cases } \\
(\mathrm{n}=999)^{\mathrm{e}}\end{array}$ & $\begin{array}{l}\text { Controls } \\
(n=1998)^{e}\end{array}$ & $\begin{array}{l}\text { CADD } \\
\text { scaledC } \\
\text { score }\end{array}$ & Condel & PolyPhen2 & SIFT \\
\hline 1 & $\mathrm{C} .11 \mathrm{C}>\mathrm{T}$ & p.Pro4Leu & rs45619737 & $\begin{array}{l}\text { Rahman } \\
\text { (2007) [3] }\end{array}$ & . & 0.00038 & 0.0000999 & 1 & 1 & 1 & 12.9 & Deleterious & $\begin{array}{l}\text { Probably } \\
\text { damaging }\end{array}$ & Tolerated \\
\hline 2 & c. $53 \mathrm{~A}>\mathrm{G}$ & p.Lys18Arg & rs138789658 & $\begin{array}{l}\text { Tischkowitz } \\
\text { (2008) }\end{array}$ & 0.0032 & 0.00523 & 0.0000552 & - & - & 1 & 18.0 & Deleterious & $\begin{array}{l}\text { Possibly } \\
\text { damaging }\end{array}$ & Deleterious \\
\hline 2 & c. $.94 C>G$ & p.Leu32Val & rs151316635 & Teo (2013) [8] & & 0.00023 & 0.0000184 & - & 1 & 1 & 16.3 & Deleterious & $\begin{array}{l}\text { Possibly } \\
\text { damaging }\end{array}$ & Deleterious \\
\hline 3 & c. $194 C>T$ & p.Pro65Leu & rs62625272 & $\begin{array}{l}\text { Adank (2011) } \\
\text { [26] }\end{array}$ & & 0.00015 & 0.00006 & - & - & 1 & 4.6 & Neutral & Benign & Tolerated \\
\hline 4 & c. $232 \mathrm{G}>\mathrm{A}$ & p.Val78lle & & $\begin{array}{l}\text { Tischkowitz } \\
\text { (2012) [39] }\end{array}$ & . & & 0.000325 & & 2 & & 0.4 & Neutral & Benign & Tolerated \\
\hline 4 & c. $298 \mathrm{C}>\mathrm{T}$ & p.Leu100Phe & rs61756147 & $\begin{array}{l}\text { Wong } \\
\text { (2011) [9] }\end{array}$ & 0.0005 & 0.00023 & 0.000037 & - & - & 2 & 10.3 & Neutral & $\begin{array}{l}\text { Probably } \\
\text { damaging }\end{array}$ & Tolerated \\
\hline 4 & c.344G > T & p.Gly115Val & rs145598272 & $\begin{array}{l}\text { Foulkes } \\
\text { (2007) [34] }\end{array}$ & & 0.00015 & 0.0000921 & 1 & - & 1 & 6.5 & Neutral & Benign & Tolerated \\
\hline 4 & c.353 T > C & p.lle118Thr & & - & . & 0.00008 & 0.000037 & - & - & 1 & 5.4 & Neutral & Benign & Tolerated \\
\hline 4 & c. $400 \mathrm{G}>\mathrm{A}$ & p.Asp134Asn & rs139555085 & Zheng (2011) & 0.0005 & 0.00184 & 0 & & & 1 & 4.8 & Neutral & Benign & Tolerated \\
\hline 4 & $c .508 \mathrm{~A}>\mathrm{G}$ & p.Arg170Gly & & - & . & & & - & - & 1 & 4.4 & Neutral & Benign & Tolerated \\
\hline 4 & c. $557 \mathrm{~A}>\mathrm{T}$ & p.Asn186lle & . & - & & . & . & - & - & 1 & 13.1 & Deleterious & $\begin{array}{l}\text { Probably } \\
\text { damaging }\end{array}$ & Deleterious \\
\hline 4 & $C .571 C>G$ & p.Pro191Ala & . & - & & . & . & - & - & 1 & 9.4 & Neutral & $\begin{array}{l}\text { Probably } \\
\text { damaging }\end{array}$ & Tolerated \\
\hline 4 & c. $629 \mathrm{C}>\mathrm{T}$ & p.Pro210Leu & rs57605939 & $\begin{array}{l}\text { Rahman } \\
\text { (2007) [3] }\end{array}$ & 0.0174 & 0.02216 & 0.0000736 & 1 & 1 & 2 & 10.6 & Deleterious & $\begin{array}{l}\text { Probably } \\
\text { damaging }\end{array}$ & Tolerated \\
\hline 4 & c. $899 \mathrm{C}>\mathrm{T}$ & p.Thr300lle & . & Ding (2011) & & . & 0 & 1 & - & - & 15.1 & Deleterious & $\begin{array}{l}\text { Probably } \\
\text { damaging }\end{array}$ & Tolerated \\
\hline 4 & c. $656 \mathrm{~A}>\mathrm{G}$ & p.Asp219Gly & rs45594034 & $\begin{array}{l}\text { Rahman } \\
\text { (2007) [3] }\end{array}$ & & 0.00015 & 0.000221 & & 1 & & 1.8 & Neutral & Benign & Tolerated \\
\hline 4 & c. $740 C>G$ & p.Thr247Arg & & & . & & & & 1 & & 15.5 & Deleterious & $\begin{array}{l}\text { Probably } \\
\text { damaging }\end{array}$ & Tolerated \\
\hline 4 & c. $.925 A>G$ & p.lle309Val & rs3809683 & $\begin{array}{l}\text { Rahman } \\
\text { (2007) [3] }\end{array}$ & 0.0087 & 0.00970 & 0.0000184 & $4(1)$ & - & - & 0.5 & Neutral & Benign & Tolerated \\
\hline 4 & c.1010 T>C & p.Leu337Ser & rs45494092 & $\begin{array}{l}\text { Rahman } \\
\text { (2007) [3] }\end{array}$ & 0.0133 & 0.01424 & 0.0197 & $44(1)$ & 44 & $93(1)$ & 8.9 & Deleterious & $\begin{array}{l}\text { Probably } \\
\text { damaging }\end{array}$ & Tolerated \\
\hline 4 & c.1085 T>C & p.Leu362Pro & . & - & & . & & 1 & - & - & 14.4 & Deleterious & $\begin{array}{l}\text { Probably } \\
\text { damaging }\end{array}$ & Tolerated \\
\hline 4 & c.1145G > T & p.Ser382lle & . & $\begin{array}{l}\text { Tischkowitz } \\
\text { (2012) [39] }\end{array}$ & & . & 0.0000184 & - & 1 & - & 15.2 & Deleterious & $\begin{array}{l}\text { Possibly_ } \\
\text { damaging }\end{array}$ & Deleterious \\
\hline
\end{tabular}


Table 3 Missense variants (Continued)

\begin{tabular}{|c|c|c|c|c|c|c|c|c|c|c|c|c|c|c|}
\hline 4 & C. $1189 \mathrm{~A}>\mathrm{T}$ & p.Thr397Ser & . & $\begin{array}{l}\text { Rahman } \\
\text { (2007) [3] }\end{array}$ & . & 0.00008 & 0.0000184 & - & 1 & 1 & 22.9 & Deleterious & $\begin{array}{l}\text { Possibly } \\
\text { damaging }\end{array}$ & Deleterious \\
\hline 4 & C. $1250 C>A$ & p.Ser417Tyr & rs45510998 & $\begin{array}{l}\text { Rahman } \\
\text { (2007) }\end{array}$ & . & & 0.000203 & - & - & 1 & 20.6 & Deleterious & $\begin{array}{l}\text { Probably } \\
\text { damaging }\end{array}$ & Deleterious \\
\hline 4 & c. $1478 \mathrm{C}>\mathrm{T}$ & p.Pro493Leu & . & - & & . & 0.0000184 & - & - & 1 & 12.4 & Neutral & Benign & Tolerated \\
\hline 4 & c. $1492 \mathrm{G}>\mathrm{T}$ & p.Asp498Tyr & rs75023630 & $\begin{array}{l}\text { Phuah } \\
\text { (2013) [38] }\end{array}$ & 0.0014 & & 0 & - & - & 1 & 16.2 & Deleterious & Benign & Deleterious \\
\hline 4 & c. $1544 A>G$ & p.Lys515Arg & . & $\begin{array}{l}\text { Tischkowitz } \\
\text { (2012) [39] }\end{array}$ & & . & 0.000037 & - & 1 & - & 16.2 & Deleterious & $\begin{array}{l}\text { Possibly } \\
\text { damaging }\end{array}$ & Tolerated \\
\hline 4 & c. $1610 C>T$ & p.Ser537Leu & rs142103232 & - & & 0.00015 & 0.000166 & - & 1 & - & 11.2 & Neutral & $\begin{array}{l}\text { Possibly } \\
\text { damaging }\end{array}$ & Tolerated \\
\hline 4 & c. $1676 A>G$ & p.Gln559Arg & rs152451 & $\begin{array}{l}\text { Rahman } \\
\text { (2007) [3] }\end{array}$ & 0.1465 & 0.13483 & 0.0961 & $216(9)$ & $182(11)$ & $335(10)$ & 0.0 & Neutral & Benign & Tolerated \\
\hline 5 & c. 1699 C > T & p.His567Tyr & & $\begin{array}{l}\text { Tischkowitz } \\
\text { (2012) [39] }\end{array}$ & . & 0.00008 & 0.000094 & - & - & 1 & 0.9 & Neutral & Benign & Tolerated \\
\hline 5 & c.1931G > A & p.Gly644Glu & . & - & & . & . & - & - & 1 & 16.0 & Deleterious & $\begin{array}{l}\text { Probably } \\
\text { damaging }\end{array}$ & Deleterious \\
\hline 5 & c. $2014 G>C$ & p.Glu672Gln & rs45532440 & $\begin{array}{l}\text { Rahman } \\
\text { (2007) [3] }\end{array}$ & 0.0142 & 0.02324 & 0.0278 & $64(1)$ & $68(4)$ & $123(1)$ & 11.4 & Neutral & $\begin{array}{l}\text { Possibly } \\
\text { damaging }\end{array}$ & Tolerated \\
\hline 5 & c. $2106 A>G$ & p.lle702Met & & - & & & 0.0000184 & 1 & - & - & 11.1 & Neutral & $\begin{array}{l}\text { Probably } \\
\text { damaging }\end{array}$ & Tolerated \\
\hline 5 & $c .2135 C>T$ & p.Ala712Val & rs141458731 & $\begin{array}{l}\text { Dansonka- } \\
\text { Meiszkowska } \\
\text { (2010) }\end{array}$ & 0.0014 & 0.00062 & 0.00039 & - & - & 1 & 12.2 & Neutral & Benign & Tolerated \\
\hline 5 & $c .2200 A>T$ & p.Thr734Ser & rs45543843 & $\begin{array}{l}\text { Rahman } \\
\text { (2007) [3] }\end{array}$ & . & & 0.0000368 & - & - & 3 & 25.3 & Deleterious & $\begin{array}{l}\text { Possibly } \\
\text { damaging }\end{array}$ & Deleterious \\
\hline 5 & c. $2228 \mathrm{~A}>\mathrm{G}$ & p.Tyr743Cys & rs 141749524 & - & 0.0009 & . & 0.0000184 & 1 & - & - & 8.5 & Neutral & Benign & Tolerated \\
\hline 5 & c. $2289 G>C$ & p.Leu763Phe & . & $\begin{array}{l}\text { Phuah } \\
\text { (2013) [38] }\end{array}$ & & . & 0 & 2 & - & - & 15.5 & Neutral & $\begin{array}{l}\text { Probably } \\
\text { damaging }\end{array}$ & Tolerated \\
\hline 5 & $c .2360 C>T$ & p.Thr787lle & rs201042302 & - & 0.0005 & & 0 & - & 1 & - & 9.7 & Neutral & $\begin{array}{l}\text { Probably } \\
\text { damaging }\end{array}$ & Tolerated \\
\hline 5 & c. $2417 C>T$ & p.Pro806Leu & rs45464991 & $\begin{array}{l}\text { Rahman } \\
\text { (2007) [3] }\end{array}$ & & 0.00008 & 0.000037 & - & 1 & 1 & 0.5 & Neutral & Benign & Tolerated \\
\hline 7 & c. $2590 \mathrm{C}>\mathrm{T}$ & p.Pro864Ser & rs45568339 & $\begin{array}{l}\text { Rahman } \\
\text { (2007) [3] }\end{array}$ & 0.0018 & 0.00239 & 0.00396 & 6 & 10 & 19 & 12.0 & Neutral & Benign & Tolerated \\
\hline 7 & c. $2606 C>G$ & p.Ser869Cys & & & . & & 0.0000184 & & 1 & & 19.9 & Deleterious & $\begin{array}{l}\text { Probably } \\
\text { damaging }\end{array}$ & Deleterious \\
\hline 7 & c. $2674 G>A$ & p.Glu892Lys & rs45476495 & $\begin{array}{l}\text { Rahman } \\
\text { (2007) [3] }\end{array}$ & & 0.00008 & 0.000092 & 1 & 2 & - & 20.8 & Deleterious & $\begin{array}{l}\text { Possibly } \\
\text { damaging }\end{array}$ & Deleterious \\
\hline 8 & $c .2755 G>A$ & p.Val919lle & & - & . & & & - & - & 1 & 15.7 & Neutral & Benign & Tolerated \\
\hline
\end{tabular}


Table 3 Missense variants (Continued)

\begin{tabular}{|c|c|c|c|c|c|c|c|c|c|c|c|c|c|c|}
\hline 8 & $c .2794 G>A$ & p.Val932Met & rs45624036 & $\begin{array}{l}\text { Rahman } \\
\text { (2007) [3] }\end{array}$ & 0.0009 & 0.00431 & 0.00869 & 15 & 8 & 23 & 18.3 & Deleterious & $\begin{array}{l}\text { Probably } \\
\text { damaging }\end{array}$ & Tolerated \\
\hline 8 & c. $2816 \mathrm{~T}>\mathrm{G}$ & p.Leu939Trp & rs45478192 & $\begin{array}{l}\text { Rahman } \\
\text { (2007) [3] }\end{array}$ & 0.0009 & 0.00154 & 0.0015 & 2 & $3(1)$ & 8 & 20.9 & Deleterious & $\begin{array}{l}\text { Probably } \\
\text { damaging }\end{array}$ & Deleterious \\
\hline 9 & c. $2993 \mathrm{G}>\mathrm{A}$ & p.Gly998Glu & rs45551636 & $\begin{array}{l}\text { Rahman } \\
\text { (2007) [3] }\end{array}$ & 0.0105 & 0.01785 & 0.0213 & 42 & $46(2)$ & $92(1)$ & 22.7 & Deleterious & $\begin{array}{l}\text { Probably } \\
\text { damaging }\end{array}$ & Deleterious \\
\hline 10 & c. $3054 \mathrm{G}>\mathrm{C}$ & p.Glu1018Asp & rs183489969 & $\begin{array}{l}\text { Tischkowitz } \\
\text { (2012) [39] }\end{array}$ & 0.0009 & . & 0 & 1 & - & 1 & 16.4 & Deleterious & $\begin{array}{l}\text { Possibly } \\
\text { damaging }\end{array}$ & Deleterious \\
\hline 10 & c. $3106 \mathrm{G}>\mathrm{C}$ & p.Val1036Leu & & - & & & 0.000037 & - & 1 & - & 13.3 & Neutral & Benign & Tolerated \\
\hline 11 & c. $3128 \mathrm{G}>\mathrm{C}$ & p.Gly1043Ala & . & $\begin{array}{l}\text { Hellebrand } \\
\text { (2011) [36] }\end{array}$ & 0.0009 & . & 0.000037 & 1 & - & - & 20.9 & Deleterious & $\begin{array}{l}\text { Probably } \\
\text { damaging }\end{array}$ & Deleterious \\
\hline 11 & c. $3146 \mathrm{~T}>\mathrm{C}$ & p.Met1049Thr & rs138273800 & - & & 0.00008 & . & - & 1 & - & 18.0 & Deleterious & $\begin{array}{l}\text { Probably } \\
\text { damaging }\end{array}$ & Deleterious \\
\hline 12 & c. $3307 \mathrm{G}>\mathrm{A}$ & p.Val1 103Met & rs201657283 & $\begin{array}{l}\text { Casadei } \\
\text { (2011) [29] }\end{array}$ & & 0.00015 & 0.0000184 & - & 1 & - & 13.0 & Neutral & Benign & Tolerated \\
\hline 13 & c. $3366 C>A$ & p.Asp1122Glu & . & . & & . & . & - & & 1 & 16.0 & Neutral & $\begin{array}{l}\text { Possibly } \\
\text { damaging }\end{array}$ & Tolerated \\
\hline 13 & c.3367G > A & p.Val1123Met & & & & . & . & - & 1 & - & 18.7 & Deleterious & $\begin{array}{l}\text { Probably } \\
\text { damaging }\end{array}$ & Deleterious \\
\hline 13 & c. $3428 \mathrm{~T}>\mathrm{A}$ & p.Leu1143His & rs62625284 & Balia (2010) & . & 0.00008 & 0.00031 & 2 & - & - & 20.0 & Deleterious & $\begin{array}{l}\text { Possibly } \\
\text { damaging }\end{array}$ & Tolerated \\
\hline 13 & c. $3448 C>T$ & p.Leu1150Phe & & & . & & & - & 1 & - & 15.5 & Deleterious & $\begin{array}{l}\text { Possibly } \\
\text { damaging }\end{array}$ & Deleterious \\
\hline 13 & c. $3449 \mathrm{~T}>\mathrm{G}$ & p.Leu1150Arg & rs45566737 & & . & . & 0.0000368 & - & 1 & - & 18.2 & Deleterious & $\begin{array}{l}\text { Possibly } \\
\text { damaging }\end{array}$ & Deleterious \\
\hline
\end{tabular}

HAPS Hunter Area Pathology Service, FCC Familial Cancer Centre, ViP Variants in Practice

avariant positions are reported in reference to NCBI RefSeq NM_024675.3 (mRNA) and NP_078951.2 (protein)

${ }^{\mathrm{b}}$ Minor allele frequency (MAF) reported in the 1000 Genomes (1000 G) cohort Phase 1

'MAF reported in the Exome Variant Server, NHLBI GO Exome Sequencing Project (ESP) [46] (data release ESP6500SI-V2)

dMAF reported in ExAC [45] from non-Finnish Europeans, excluding individuals in the database who were part of The Cancer Genome Atlas and therefore known to have had cancer

eNumber of individuals carrying the variant. Where applicable, the number of homozygous carriers is indicated in parentheses 
Table 4 Synonymous variants

\begin{tabular}{|c|c|c|c|c|c|c|c|c|c|c|c|}
\hline Exon & $\begin{array}{l}\text { Nucleotide } \\
\text { change }^{a}\end{array}$ & $\begin{array}{l}\text { Protein } \\
\text { change }^{a}\end{array}$ & dbSNP ID & First reported & $\begin{array}{l}1000 \mathrm{G} \\
\mathrm{MAF}^{\mathrm{b}}\end{array}$ & $\begin{array}{l}\text { NHBLI GO } \\
\text { ESP MAF }^{c}\end{array}$ & ExAC $^{d}$ & $\begin{array}{l}\text { HAPS cases } \\
(n=997)^{e}\end{array}$ & $\begin{array}{l}\text { FCC-ViP } \\
\text { cases } \\
(n=999)^{d}\end{array}$ & $\begin{array}{l}\text { Controls } \\
(n=1998)^{e}\end{array}$ & $\begin{array}{l}\text { CADD } \\
\text { scaledC } \\
\text { score }\end{array}$ \\
\hline 1 & $\mathrm{c} .12 \mathrm{~T}>\mathrm{C}$ & p. $(=)$ & rs145291423 & - & . & . & 0.00012 & - & 1 & 1 & 11.2 \\
\hline 4 & c. $768 \mathrm{C}>\mathrm{T}$ & p. $(=)$ & rs45487491 & & . & . & 0.000037 & & 1 & & 0.3 \\
\hline 4 & C. $1188 \mathrm{C}>\mathrm{T}$ & p. $(=)$ & . & - & . & . & 0.00003 & - & - & 1 & 7.6 \\
\hline 4 & C.1194G > A & p. $(=)$ & rs61755173 & Rahman (2007) [3] & 0.0009 & 0.00154 & 0.00114 & 3 & 2 & 8 & 6.4 \\
\hline 4 & c.1194G > T & p. $(=)$ & . & - & . & . & . & 1 & - & - & 6.1 \\
\hline 4 & C. $1242 A>C$ & p. $(=)$ & . & - & . & . & . & - & - & 1 & 7.7 \\
\hline 4 & c. $1431 C>T$ & p. $(=)$ & & Teo (2013) & & & 0 & 2 & - & - & 0.0 \\
\hline 4 & c. $1470 \mathrm{C}>\mathrm{T}$ & p. $(=)$ & rs45612837 & Rahman (2007) [3] & 0.0005 & 0.00015 & 0.000405 & 1 & - & 2 & 5.8 \\
\hline 4 & c. $1572 A>G$ & p. $(=)$ & rs45472400 & Rahman (2007) [3] & 0.0032 & 0.00339 & 0.0041 & 12 & 12 & 27 & 5.6 \\
\hline 4 & c.1623G > A & p. $(=)$ & . & - & r. & . & 0 & - & 1 & 2 & 4.2 \\
\hline 5 & c. $2067 \mathrm{G}>\mathrm{A}$ & p. $(=)$ & . & Phuah (2013) [38] & . & 0.00015 & 0.000018 & 1 & - & - & 4.1 \\
\hline 5 & c. $2082 A>G$ & p. $(=)$ & rs150569240 & - & . & . & 0.000055 & - & - & 1 & 5.8 \\
\hline 5 & c. $2091 C>A$ & p. $(=)$ & . & - & . & . & 0 & - & - & 1 & 4.7 \\
\hline 5 & c. $2244 A>G$ & p. $(=)$ & & - & & & 0.000037 & - & - & 1 & 5.6 \\
\hline 5 & c. $2328 \mathrm{C}>\mathrm{T}$ & p. $(=)$ & rs45508997 & - & . & 0.00008 & 0 & 1 & - & - & 4.0 \\
\hline 5 & c. $2337 A>C$ & p. $(=)$ & . & - & . & . & & - & 1 & - & 5.2 \\
\hline 5 & c. $2379 C>T$ & p. $(=)$ & . & - & . & 0.00008 & 0.000111 & 1 & 1 & - & 0.1 \\
\hline 5 & c. $2478 C>T$ & p. $(=)$ & & - & & & . & - & - & 1 & 4.2 \\
\hline 5 & c. $2484 C>T$ & p. $(=)$ & . & - & . & . & . & - & - & 1 & 6.0 \\
\hline 7 & c. $2742 C>T$ & p. $(=)$ & rs115759702 & - & 0.0018 & 0.00146 & 0.00003 & 2 & - & - & 0.7 \\
\hline 12 & c.3294G > A & p. $(=)$ & . & - & . & . & . & - & - & 1 & 9.9 \\
\hline 12 & c.3300 T>G & p. $(=)$ & rs45516100 & Rahman (2007) [3] & 0.0183 & 0.02801 & 0.0278 & $64(1)$ & $68(4)$ & $123(1)$ & 7.9 \\
\hline 13 & c. $3495 \mathrm{G}>\mathrm{A}$ & p. $(=)$ & rs45439097 & Bogdanova (2010) & & 0.00108 & 0.000994 & 4 & 7 & 9 & 8.6 \\
\hline
\end{tabular}

HAPS Hunter Area Pathology Service, FCC Familial Cancer Centre, ViP Variants in Practice

avariant positions are reported in reference to NCBI RefSeq NM 024675.3 (mRNA) and NP 078951.2 (protein)

${ }^{\mathrm{b}}$ Minor allele frequency (MAF) reported in the 1000 Genomes $(1000 \mathrm{G})$ cohort Phase 1

'MAF reported in the Exome Variant Server, NHLBI GO Exome Sequencing Project (ESP) [46] (data release ESP6500SI-V2)

${ }^{\mathrm{d} M A F}$ reported in ExAC [45] from non-Finnish Europeans, excluding individuals in the database who were part of The Cancer Genome Atlas and therefore known to have had cancer

${ }^{\mathrm{e}}$ Number of individuals carrying the variant. Where applicable, the number of homozygous carriers is indicated in parentheses

controls will be required to confirm whether the association of rs152451 with breast cancer is a robust finding. In addition, the wide variation in the frequency of the minor allele in different populations means that cases and controls will have to be carefully matched for ethnicity. This variant is not located in a known protein domain and was consistently found to have predicted benign effects on protein function by all algorithms tested. However, this base change is only 9 bp away from the exon 4 splice donor site and Human Splicing Finder (v3) found that rs152451 could alter an exonic splicing enhancer motif [48], offering a potential mechanism for how this variant could affect PALB2 function. It should be noted that such a prediction was relatively common for the variants we detected in PALB2 (35/77 missense or nonsynonymous variants had a similar prediction from at least three algorithms) and any effect would need to be confirmed by an RNA-based assay.

There has been only one study to date that has examined the likely functional effect of missense variants in PALB2, which examined p.Leu939Trp, p.Leu1143Pro and p.Thr1030Ile [49]. The first two variants had subtle but significant effects on homologous recombination repair: p.Leu1143Pro in particular showed decreased repair capacity and binding to BRCA2 and RAD51C. PALB2 p.Thr1030Ile was unstable, leading to decreased protein levels and this was assumed to impair homologous recombination repair. However, it should be noted that these functional assays were performed by overexpression of a retroviral transgene in a null cell line and may not reflect the heterozygote situation. In our study, p.Leu939Trp was not enriched in cases (four in cases, eight in controls), 
p.Leu1143Pro was only seen in two cases and no controls, while p.Thr1030Ile was not observed in either cases or controls.

\section{Conclusions}

Our data strongly support PALB2 as a breast cancer predisposition gene when considering truncating mutations. We did not see any excess in missense mutations in cancer cases overall, although there may be individual variants that are associated with risk at low penetrance. We advise extreme caution in attributing risk to missense PALB2 mutations when determining clinical management.

\section{Additional files}

Additional file 1: Cohort information (Table). (DOCX $48 \mathrm{~kb}$ )

Additional file 2: PALB2 truncating variant carrier family pedigrees (Figure). (PDF $1036 \mathrm{~kb}$ )

\section{Abbreviations}

Cl: confidence interval; FCC: Familial Cancer Centre; HAPS: Hunter Area Pathology Service; OR: odds ratio; ViP: Variants in Practice.

\section{Competing interests}

The authors declare that they have no competing interests.

\section{Authors' contributions}

IGC, PAJ, Lifepool Investigators, RJS, AHT and GM conceived of and designed the study. ERT, NL, LD, JL, RL, MAD, MWB, MBD, SM, PAJ and SMR carried out experiments, acquired and analysed data. MWB, LD, SM, RJS, MBD, Lifepool Investigators, AHT and PAJ provided data and samples. KLG interpreted data. IGC, PAJ, GM, KLG, SMR, RJS and ERT were involved in drafting the manuscript and all authors read and provided critical feedback on the manuscript. All authors had final approval of the submitted and published versions and are accountable for the work.

\section{Acknowledgements}

We would like to thank all centres who provided samples for BRCA1 and $B R C A 2$ analysis to HAPS, which were used in this study and the investigators/ FCCS of the ViP study: Marion Harris (Monash Health), Geoff Lindeman (Royal Melbourne Hospital), Martin Delatycki (Austin Hospital), Yoland Antill (Cabrini Hospital) and Ingrid Winship (Geelong FCC/Barwon Health). Ethics approval for the ViP study was provided from all the above-listed centres. Lifepool Investigators (lifepool@petermac.org; LifePool, Peter MacCallum Cancer Centre, East Melbourne, Victoria, Australia) include lan Campbell, John Hopper, Vicki Pridmore, Anne Kavanagh, Gillian Mitchell, Bruce Mann and Stephen Fox.

This work was supported by the Victorian Breast Cancer Research Consortium, the National Breast Cancer Foundation, the Victorian Cancer Agency, and the National Health and Medical Research Council.

\footnotetext{
Author details

${ }^{1}$ Cancer Genetics Laboratory, Peter MacCallum Cancer Centre, Locked Bag 1, A'Beckett St, East Melbourne, VIC 8006, Australia. ${ }^{2}$ The Sir Peter MacCallum Department of Oncology, St Andrews Place, East Melbourne, VIC 3002, Australia. ${ }^{3}$ Department of Pathology, University of Melbourne, Corner GrattonStree and Royal Parade, Melbourne, VIC 3010, Australia. ${ }^{4}$ Discipline of Medical Genetics and Centre for Information-Based Medicine, The University of Newcastle and Hunter Medical Research Institute, 1 Kookaburra Circuit, Newcastle, NSW 2305, Australia. ${ }^{5}$ Familial Cancer Centre, Peter MacCallum Cancer Centre, St Andrews Place, East Melbourne, VIC 3002, Australia. ${ }^{6}$ Cancer Biology Research Center, Tongji Hospital, Tongji Medical College, Huazhong University of Science and Technology, 1037 Luoyu Road, Wuhan, Hubei, China. 'LifePool, Peter MacCallum Cancer Centre, St Andrews Place,
}

East Melbourne, VIC 3002, Australia. ${ }^{8}$ Bioinformatics Core Facility, Peter MacCallum Cancer Centre, St Andrews Place, East Melbourne, VIC 3002 Australia. ${ }^{9}$ Austin Health, 145 Studley Road, Heidelberg, VIC 3084, Australia. ${ }^{10}$ Hereditary Cancer Program, BC Cancer Agency, 600 West 10th Avenue, Vancouver, BC V5Z 4E6, Canada. ${ }^{11}$ Division of Genetics, Hunter Area Pathology Service, Lookout Road, Newcastle, NSW 2305, Australia.

Received: 15 May 2015 Accepted: 4 August 2015

Published online: 19 August 2015

\section{References}

1. Xia B, Dorsman JC, Ameziane N, de Vries Y, Rooimans MA, Sheng Q, et al. Fanconi anemia is associated with a defect in the BRCA2 partner PALB2. Nat Genet. 2007;39:159-61. doi:10.1038/ng1942.

2. Reid S, Schindler D, Hanenberg H, Barker $K$, Hanks S, Kalb R, et al. Biallelic mutations in PALB2 cause Fanconi anemia subtype FA-N and predispose to childhood cancer. Nat Genet. 2007:39:162-4. doi:10.1038/ng1947.

3. Rahman N, Seal S, Thompson D, Kelly P, Renwick A, Elliott A, et al. PALB2, which encodes a BRCA2-interacting protein, is a breast cancer susceptibility gene. Nat Genet. 2007;39:165-7. doi:10.1038/ng1959.

4. Southey MC, Teo ZL, Winship I. PALB2 and breast cancer: ready for clinical translation! Appl Clin Genet. 2013:6:43-52. doi:10.2147/TACG.S34116.

5. Antoniou AC, Casadei S, Heikkinen T, Barrowdale D, Pylkas K, Roberts J, et al. Breast-cancer risk in families with mutations in PALB2. N Engl J Med. 2014;371:497-506. doi:10.1056/NEJMoa1400382.

6. Southey MC, Teo ZL, Dowty JG, Odefrey FA, Park DJ, Tischkowitz M, et al. A PALB2 mutation associated with high risk of breast cancer. Breast Cancer Res. 2010;12:R109. doi:10.1186/bcr2796.

7. Nguyen-Dumont T, Hammet F, Mahmoodi M, Tsimiklis H, Teo ZL, Li R, et al. Mutation screening of PALB2 in clinically ascertained families from the Breast Cancer Family Registry. Breast Cancer Res Treat. 2015;149:547-54. doi:10.1007/s10549-014-3260-8.

8. Teo ZL, Park DJ, Provenzano E, Chatfield CA, Odefrey FA, Nguyen-Dumont T, et al. Prevalence of PALB2 mutations in Australasian multiple-case breast cancer families. Breast Cancer Res. 2013;15:R17. doi:10.1186/bcr3392.

9. Wong MW, Nordfors C, Mossman D, Pecenpetelovska G, Avery-Kiejda KA, Talseth-Palmer B, et al. BRIP1, PALB2, and RAD51C mutation analysis reveals their relative importance as genetic susceptibility factors for breast cancer. Breast Cancer Res Treat. 2011;127:853-9. doi:10.1007/s10549-011-1443-0.

10. Wong-Brown MW, Avery-Kiejda KA, Bowden NA, Scott RJ. Low prevalence of germline PALB2 mutations in Australian triple-negative breast cancer. Int J Cancer. 2014;134:301-5. doi:10.1002/ijc.28361.

11. EviQ cancer treatments online http://www.eviq.org.au. Accessed May 2015

12. National Comprehensive Cancer Network http://www.nccn.org/ professionals/physician_gls/pdf/genetics_screening.pdf. Accessed May 2015

13. Sawyer S, Mitchell G, McKinley J, Chenevix-Trench G, Beesley J, Chen XQ, et al. A role for common genomic variants in the assessment of familial breast cancer. J Clin Oncol. 2012;30:4330-6. doi:10.1200/JCO.2012.41.7469.

14. Lifepool http://www.lifepool.org. Accessed May 2015

15. Martin M. Cutadapt removes adapter sequences from high-throughput sequencing reads. EMBnet J. 2011;17:10-2. doi:10.14806/ej.17.1.200.

16. Li H, Durbin R. Fast and accurate short read alignment with Burrows-Wheeler transform. Bioinformatics. 2009;25:1754-60. doi:10.1093/bioinformatics/btp324.

17. DePristo MA, Banks E, Poplin R, Garimella KV, Maguire JR, Hartl C, et al. A framework for variation discovery and genotyping using next-generation DNA sequencing data. Nat Genet. 2011;43:491-8. doi:10.1038/ng.806.

18. McKenna A, Hanna M, Banks E, Sivachenko A, Cibulskis K, Kernytsky A, et al. The Genome Analysis Toolkit: a MapReduce framework for analyzing nextgeneration DNA sequencing data. Genome Res. 2010;20:1297-303. doi:10.1101/gr.107524.110.

19. McLaren W, Pritchard B, Rios D, Chen Y, Flicek P, Cunningham F. Deriving the consequences of genomic variants with the Ensembl API and SNP Effect Predictor. Bioinformatics. 2010;26:2069-70. doi:10.1093/bioinformatics/btq330.

20. Human Genome Variation Society http://www.hgvs.org/mutnomen. Accessed May 2015

21. Tischkowitz M, Xia B, Sabbaghian N, Reis-Filho JS, Hamel N, Li G, et al. Analysis of PALB2/FANCN-associated breast cancer families. Proc Natl Acad Sci U S A. 2007;104:6788-93. doi:10.1073/pnas.0701724104.

22. Kircher M, Witten DM, Jain P, O'Roak BJ, Cooper GM, Shendure J. A general framework for estimating the relative pathogenicity of human genetic variants. Nat Genet. 2014;46:310-5. doi:10.1038/ng.2892. 
23. Gonzalez-Perez A, Lopez-Bigas N. Improving the assessment of the outcome of nonsynonymous SNVs with a consensus deleteriousness score. Condel Am J Hum Genet. 2011;88:440-9. doi:10.1016/j.ajhg.2011.03.004.

24. Kumar P, Henikoff S, Ng PC. Predicting the effects of coding nonsynonymous variants on protein function using the SIFT algorithm. Nat Protoc. 2009;4:1073-81. doi:10.1038/nprot.2009.86.

25. Adzhubei IA, Schmidt S, Peshkin L, Ramensky VE, Gerasimova A, Bork P, et al. A method and server for predicting damaging missense mutations. Nat Methods. 2010;7:248-9. doi:10.1038/nmeth0410-248.

26. Adank MA, van Mil SE, Gille JJ, Waisfisz Q, Meijers-Heijboer H. PALB2 analysis in BRCA2-like families. Breast Cancer Res Treat. 2011;127:357-62. doi:10.1007/s10549-010-1001-1.

27. Blanco A, de la Hoya M, Osorio A, Diez O, Miramar MD, Infante M, et al. Analysis of PALB2 gene in BRCA1/BRCA2 negative Spanish hereditary breast/ovarian cancer families with pancreatic cancer cases. PLoS One. 2013;8:e67538. doi:10.1371/journal.pone.0067538.

28. Bogdanova N, Sokolenko AP, lyevleva AG, Abysheva SN, Blaut M, Bremer M, et al. PALB2 mutations in German and Russian patients with bilateral breast cancer. Breast Cancer Res Treat. 2011;126:545-50. doi:10.1007/s10549-010-1290-4.

29. Casadei S, Norquist BM, Walsh T, Stray S, Mandell JB, Lee MK, et al. Contribution of inherited mutations in the BRCA2-interacting protein PALB2 to familial breast cancer. Cancer Res. 2011;71:2222-9. doi:10.1158/00085472.CAN-10-3958.

30. Castera L, Krieger S, Rousselin A, Legros A, Baumann JJ, Bruet O, et al. Nextgeneration sequencing for the diagnosis of hereditary breast and ovarian cancer using genomic capture targeting multiple candidate genes. Eur J Hum Genet. 2014;22:1305-13. doi:10.1038/ejhg.2014.16.

31. Catucci I, Peterlongo P, Ciceri S, Colombo M, Pasquini G, Barile M, et al. PALB2 sequencing in Italian familial breast cancer cases reveals a high-risk mutation recurrent in the province of Bergamo. Genet Med. 2014;16:688-94. doi:10.1038/gim.2014.13.

32. Erkko H, Xia B, Nikkila J, Schleutker J, Syrjakoski K, Mannermaa A, et al. A recurrent mutation in PALB2 in Finnish cancer families. Nature. 2007:446:316-9. doi:10.1038/nature05609.

33. Fernandes PH, Saam J, Peterson J, Hughes E, Kaldate R, Cummings S, et al. Comprehensive sequencing of PALB2 in patients with breast cancer suggests PALB2 mutations explain a subset of hereditary breast cancer. Cancer. 2014;120:963-7. doi:10.1002/cncr.28504.

34. Foulkes WD, Ghadirian P, Akbari MR, Hamel N, Giroux S, Sabbaghian N, et al. Identification of a novel truncating PALB2 mutation and analysis of its contribution to early-onset breast cancer in French-Canadian women. Breast Cancer Res. 2007:9:R83. doi:10.1186/bcr1828

35. Garcia MJ, Fernandez V, Osorio A, Barroso A, Llort G, Lazaro C, et al. Analysis of FANCB and FANCN/PALB2 fanconi anemia genes in BRCA1/2-negative Spanish breast cancer families. Breast Cancer Res Treat. 2009;113:545-51. doi:10.1007/s10549-008-9945-0.

36. Hellebrand H, Sutter C, Honisch E, Gross E, Wappenschmidt B, Schem C, et al. Germline mutations in the PALB2 gene are population specific and occur with low frequencies in familial breast cancer. Hum Mutat. 2011;32:E2176-88. doi:10.1002/humu.21478.

37. Janatova M, Kleibl Z, Stribrna J, Panczak A, Vesela K, Zimovjanova M, et al. The PALB2 gene is a strong candidate for clinical testing in BRCA1- and BRCA2-negative hereditary breast cancer. Cancer Epidemiol Biomarkers Prev. 2013;22:2323-32. doi:10.1158/1055-9965.EPI-13-0745-T.

38. Phuah SY, Lee SY, Kang P, Kang IN, Yoon SY, Thong MK, et al. Prevalence of PALB2 mutations in breast cancer patients in multi-ethnic Asian population in Malaysia and Singapore. PLoS One. 2013;8:e73638. doi:10.1371/ journal.pone.0073638.

39. Tischkowitz M, Capanu M, Sabbaghian N, Li L, Liang X, Vallee MP, et al. Rare germline mutations in PALB2 and breast cancer risk: a population-based study. Hum Mutat. 2012;33:674-80. doi:10.1002/humu.22022.

40. Zheng Y, Zhang J, Niu Q, Huo D, Olopade Ol. Novel germline PALB2 truncating mutations in African American breast cancer patients. Cancer. 2012;118:1362-70. doi:10.1002/cncr.26388.

41. Cybulski C, Kluzniak W, Huzarski T, Wokolorczyk D, Kashyap A, Jakubowska A, et al. Clinical outcomes in women with breast cancer and a PALB2 mutation: a prospective cohort analysis. Lancet Oncol. 2015;16:638-44. doi:10.1016/S1470-2045(15)70142-7

42. Couch FJ, Hart SN, Sharma P, Toland AE, Wang X, Miron P, et al. Inherited mutations in 17 breast cancer susceptibility genes among a large triple- negative breast cancer cohort unselected for family history of breast cancer. J Clin Oncol. 2015:33:304-11. doi:10.1200/JCO.2014.57.1414.

43. Pern F, Bogdanova N, Schurmann P, Lin M, Ay A, Langer F, et al. Mutation analysis of BRCA1, BRCA2, PALB2 and BRD7 in a hospital-based series of German patients with triple-negative breast cancer. PLoS One. 2012;7:e47993. doi:10.1371/journal.pone.0047993.

44. Genomes Project C, Abecasis GR, Auton A, Brooks LD, DePristo MA, Durbin RM, et al. An integrated map of genetic variation from 1,092 human genomes. Nature. 2012;491:56-65. doi:10.1038/nature11632.

45. Exome Aggregation Consortium http://exac.broadinstitute.org. Accessed May 2015

46. Exome Variant Server http://evs.gs.washington.edu/EVS/. Accessed May 2015

47. Leyton Y, Gonzalez-Hormazabal P, Blanco R, Bravo T, Fernandez-Ramires R, Morales $S$, et al. Association of PALB2 sequence variants with the risk of familial and early-onset breast cancer in a South-American population. BMC Cancer. 2015;15:30. doi:10.1186/s12885-015-1033-3.

48. Desmet FO, Hamroun D, Lalande M, Collod-Beroud G, Claustres M, Beroud C. Human Splicing Finder: an online bioinformatics tool to predict splicing signals. Nucleic Acids Res. 2009;37:e67. doi:10.1093/nar/gkp215.

49. Park JY, Singh TR, Nassar N, Zhang F, Freund M, Hanenberg H, et al. Breast cancer-associated missense mutants of the PALB2 WD40 domain, which directly binds RAD51C, RAD51 and BRCA2, disrupt DNA repair. Oncogene. 2014;33:4803-12. doi:10.1038/onc.2013.421.

50. Jones S, Hruban RH, Kamiyama M, Borges M, Zhang X, Parsons DW, et al. Exomic Sequencing Identifies PALB2 as a Pancreatic Cancer Susceptibility Gene. Science. 2009;324(5924):217. doi:10.1126/science.1171202.

51. Wong-Brown MW, Avery-Kiejda KA, Bowden NA, Scott RJ. Low prevalence of germline PALB2 mutations in Australian triple-negative breast cancer. Int J Cancer. 2014;134:301-305.

52. Tischkowitz M, Sabbaghian N, Ray AM, Lange EM, Foulkes WD, Cooney KA. Analysis of the Gene Coding for the BRCA2-Interacting Protein PALB2 in Hereditary Prostate Cancer. Prostate. 2008;68(6):675-678. doi:10.1002/pros.20729.

53. Zheng Y, Zhang J, Niu Q, Huo D, Olopade Ol. Novel germline PALB2 truncating mutations in African-American breast cancer patients. Cancer. 2012;118(5):1362-1370. doi:10.1002/cncr.26388.

54. Ding YC, Steele L, Chu L-H, Kelley K, Davis H, John EM, et al. Germline mutations in PALB2 in African-American breast cancer cases. Breast Cancer Res Treat. 2011;126(1):227-230. doi:10.1007/s10549-010-1271-7.

55. Dansonka-Mieszkowska A, Kluska A, Moes J, Dabrowska M, Nowakowska D, Niwinska A, et al. A novel germline PALB2 deletion in Polish breast and ovarian cancer patients. BMC Med Genet. 2010;11:20. http://www.biomedcentral.com/ 1471-2350/11/20.

56. Balia C, Sensi E, Lombardi G, Roncella M, Bevilacqua G, Caligo MA. PALB2: a novel inactivating mutation in a Italian breast cancer family. Fam Cancer. 2010;9:531-536. doi:10.1007/s10689-010-9382-1.

57. Bogdanova N, Sokolenko AP, lyevleva AG, Abysheva SN, Blaut M, Bremer M, et al. PALB2 mutations in German and Russian patients with bilateral breast cancer. Breast Cancer Res Treat. 2011;126:545-550. doi:10.1007/s10549-010-1290-4. 